\title{
Signaling, Random Assignment, and Causal Effect Estimation*
}

\author{
Christopher A. Hennessy \\ Gilles Chemla \\ LBS, CEPR, and ECGI. \\ Imperial College, DRM/CNRS, and CEPR.
}

July 2020

\begin{abstract}
Causal evidence from random assignment has been labeled "the most credible." We argue it is generally incomplete in finance/economics, omitting central parts of the true empirical causal chain. Random assignment, in eliminating self-selection, simultaneously precludes signaling via treatment choice. However, outside experiments, agents enjoy discretion to signal, thereby causing changes in beliefs and outcomes. Therefore, if the goal is informing discretionary decisions, rather than predicting outcomes after forced/mistaken actions, randomization is problematic. As shown, signaling can amplify, attenuate, or reverse signs of causal effects. Thus, traditional methods of empirical finance, e.g. event studies, are often more credible/useful.
\end{abstract}

\section{Introduction}

It is hard to overstate the influence of Angrist and Pischke's (2009) Mostly Harmless Econometrics on modern-day empirical finance. Even the language of the profession has shifted, as evidenced by frequent use of the terms "identification" and "causal effect." As a lagging indicator, Bowen, Frésard, and Taillard (2016) find that in the top-three finance journals, the share of empirical corporate finance papers using what they term "identification technologies" rose from roughly 0 percent in the late 1980's to over 50 percent by 2012. As an example of the converse, consider that in 1986 the Journal of Financial Economics devoted one-half of a double issue to five event

\footnotetext{
${ }^{*}$ Contact chennessy@london.edu (corresponding author) and g.chemla@imperial.ac.uk. We thank seminar participants at Université Paris Dauphine, LBS, Imperial College, and ESCP Europe.
} 
studies analyzing endogenous capital structure changes, an unlikely journal configuration given contemporary methodological norms.

The crowding-out of such quasi-observational studies is understandable, and indeed a good thing for the progress of finance as a science, if one accepts the initial premise put forward by Angrist and Pischke (2009) that, "The goal of most empirical research is to overcome selection bias, and therefore to have something to say about the causal effect of a variable." That is, according to the working definition of Angrist and Pischke (2009), selection effects are decidedly not an element of causal effects. To the contrary, selection effects mask true causal effects. If this definition of causal effect is accepted, then it must be agreed that, as they assert, "The most credible and influential research designs use random assignment." Moreover, as a corollary, Angrist and Pischke would have financial economists treat event studies and other traditional forms of observational evidence as being either less credible at best, or not credible at worst.

This methodological stance draws much of its inspiration from the notion that economists should strive to utilize the same causal measures and methods as medical science, an appealing metaphor at face value. For example, Duflo (2004) argues, "Creating a culture in which rigorous randomized evaluations are promoted, encouraged, and financed has the potential to revolutionize social policy during the 21st century, just as randomized trials revolutionized medicine during the 20th." Indeed, the two textbooks by Angrist and Pischke (2009, 2015) open with motivating examples of causal effects of hospitalization and insurance on health outcomes. Similarly, the influential textbook of Imbens and Rubin (2015) uses the same causal effect definition and methodological tool-kit across biomedical and social sciences.

The objective of this paper is to explore the merits and limitations of the causal effect definition and estimators as advocated by Angrist and Pischke (2009, 2015), amongst others, within the specific context of finance and economics-as distinct from medicine. To begin, we note that. to the best of our knowledge, absent from the methodological discussions and debates amongst financial economists is the fact that medical outcome variables differ in-kind from most of the financial 
outcome variables we study. In particular, true objective health outcomes are not mediated by the beliefs of other agents. ${ }^{1}$ In stark contrast, for the overwhelming majority of economic and financial variables, equilibrium outcomes are profoundly shaped by the beliefs of other financial market participants.

Should financial economists strive to attain the same type of evidence as medical scientists? Alternatively, are the qualitative differences between health outcome variables and economic outcome variables sufficiently important for financial economists to reconsider how we define and measure causal effect? In order to address these important questions, or at least initiate a more transparent and constructive dialogue on these questions, this paper analyzes the meaning and utilization of alternative causal effect measures in the types of settings commonplace in finance and economics, those where individual agents have private information and outcome variables are mediated by the beliefs of other agents in the economy.

As we show, the narrow causal effect definition utilized by Angrist and Pischke (2009, 2015), while appropriate for health outcomes, is problematic from the perspective of financial economists. This is because random assignment, in eliminating self-selection, simultaneously prevents signaling via treatment choice. After all, if a treatment is randomly forced upon an agent, the act of taking it cannot signal private information. In causal biomedical research, shutting down the signaling channel through random assignment is of no concern since an individual cannot improve their true objective health outcome by publicly and voluntarily taking a particular pill that signals something to onlookers. In sharp contrast, in a wide range of markets, individuals and firms can and do improve their objective economic and financial outcomes by publicly and voluntarily taking particular actions.

The fundamental differences between health outcomes versus economic and financial outcomes can be phrased in terms of the potential outcomes framework of Rubin (1974), a key buildingblock of modern causal inference. To begin, note that with objective health, it does not matter whether a treatment is taken under forced randomization or under individual discretion. In stark

\footnotetext{
${ }^{1}$ Health may be mediated by own-beliefs. Philipson and Desimone (1997) and Chemla and Hennessy (2019) show RCTs may deliver biased estimates of physiological effects.
} 
contrast, economic and financial outcomes often differ fundamentally according to whether an action is forced or discretionary since discretion generates signaling effects. Further, for many financial and economic variables, in contrast to health, actions that signal actually have the power to cause changes in outcomes by changing other agents' beliefs. Importantly, the signal component of the causal mechanism would even be manifest after "counterfactual" actions off the equilibrium path. For example, in a standard separating equilibrium, a CEO who knows she has low ability can nevertheless cause her company's share price to increase if she mimics high ability CEOs by purchasing a large company stake. In reality, some low-ability CEOs do just this. In stark contrast, a person with coronary disease cannot replicate the measured outcomes of the healthy simply by mimicking their behavior and avoiding medical care-quite the contrary. These fundamental differences explain why it would be foolish for an ill person to rely upon raw observational evidence whereas CEOs can, in many instances, safely rely upon "endogeneity-plagued" event studies in making decisions.

If the goal is guiding discretionary decisions, rather than predicting outcomes after forced actions or mistakes, random assignment is problematic precisely because it strips out the signal component of the overall causal mechanism. As we show, taken in isolation, estimates derived from random assignment are faulty guides regarding discretionary policies-the very types of policies that empirical evidence is often intended to inform. In fact, as shown, causal effects derived from random assignment can undershoot, overshoot, and even have signs opposite to causal effects cum signaling effects.

Undoubtedly, our understanding of causal inference has become much more nuanced over the last decade. Nevertheless, to make further progress, we suggest financial economists adopt and utilize two distinct causal effect definitions, with each being more or less useful depending on the context and decision-margin. Partial causal effects are to be understood as changes in the outcome variable arising from changes in the forcing variable holding fixed the beliefs of other agents. This type of causal effect is recovered by treatment-control differences under random assignment. As we show, partial causal effects are, perhaps surprisingly, particularly informative about deep technological 
parameters typically associated with structural inference. Moreover, if the government does not have private information, but firms do, then partial causal effects, and random assignment studies, are informative about the desirability of government-mandated policies.

Total causal effects are changes in the outcome variable resulting from discretionary changes in the forcing variable allowing for endogenous equilibrium changes in beliefs and payoffs. That is, the total causal effect is the sum of the partial causal effect and signaling effect. This causal effect measure is recovered by evidence such as announcement effects, as pioneered by Fama, Fisher, Jensen and Roll (1969). As we show, the total causal effect is often a sufficient statistic for optimal discretionary corporate decisions. Nevertheless, in some cases it may be of operational utility, and independent scientific interest, to estimate both partial and total causal effects. In fact, we illustrate how the two estimators/estimates would need to be used sequentially in order to implement optimal policy in some settings.

We clarify the issues by way of three examples. We begin with a simple yet stark example where the total causal effect is actually opposite in sign to the partial causal effect. In particular, we consider a CFO seeking to make optimal investment decisions. Here, a traditional corporate finance event study would deliver a sufficient statistic for optimality. In contrast, reliance on estimates derived from random assignment would lead to excessive investment levels. The underlying message here is that papers using some of the "outdated" methods of empirical corporate finance may well have greater value to practitioners.

Next we consider a question from household finance, e.g. Becker (1993), Roussanov (2010), and Athreya and Eberly (2020): "Are the net returns to educational investments positive?" Of course, this is a topical question presently being posed by many prospective undergraduate and MBA students. For this purpose, we extend the framework of Spence (1973) by incorporating competitive tuition-setting universities in a quantitative example. Here we illustrate how the failure to incorporate signaling benefits might understate returns by an order of magnitude. More importantly, we show this downward bias worsens as the costs of delivering education rise. This is because, as shown, 
with endogenous ability signaling, the education-wage schedule becomes steeper with higher costs of education production. Thus, the "cleanest" empirical work on returns to education, that which exploits quasi-random assignment, will understate total returns by a wide margin, and most severely, in times such as the present when rising educational production costs spur empirical enquiry.

In the final example, we consider a government with private information about economic fundamentals that is contemplating changing some policy, e.g. regulation or tax policy, in response to this information. Here we show that evidence from random assignment can identify potentially stimulative policies-in the sense of having positive partial causal effects. We then show how such analysis can complement total causal effect estimates in order to ultimately arrive at optimal policies. Thus, we conclude that in many applied settings both forms of causal effect estimates have a place in the decision-maker's tool-kit.

Our paper is related to the literature on signaling, with Spence (1973) being the pioneering paper. Ross (1977) and Leland and Pyle (1977) were early applications of signaling theory to corporate finance. For surveys, see Riley (2001) and Löfgren, Persson, and Weibull (2002). Our paper complements this literature by flushing out the implications of signaling for applied econometric work that seeks to inform decision-making by individuals, firms, and governments.

The present paper shares with Gomes (2001), Alti (2003), and Moyen (2004) the idea of using canonical models to shed light on the meaning and interpretation of empirical evidence. However, none of these papers comments on random assignment, causal effect measures, or the signaling channel. Keane (2010) and Rust (2010) argue for the need to isolate distinct causal channels and identify deep technological parameters, recommending the use of structural models for this purpose. Instead, our paper discusses how different forms of reduced-form evidence can be used to measure partial and total causal effects. There is nothing in our argument that has direct bearing on the structural versus reduced-form debate.

The central argument in our paper is most closely related to a recent paper by Fudenberg and Levine (2020). They show that Bayesian learning by agents can drive a wedge between partial 
causal effects and causal estimates derived from regression discontinuity designs. This is because uninformed agents on opposing sides of regression discontinuity boundaries endogenously form sharply different beliefs about effort returns. The central difference between the papers concerns the methodological message. In particular, Fudenberg and Levine (2020) do not challenge the primacy of partial causal effects, but rather show that standard methods may fail to recover them. In contrast, we show that when a privately informed agent moves first, partial causal effects are often a faulty basis for decision-making. Despite this difference, the papers share the notion of decomposing causal effects into (our terminology) partial causal effects and information causal effects. In their model, the information causal effect arises from the uninformed agent learning. In our model it arises from privately-informed agents signaling.

The remainder of the paper proceeds as follows. Section 2 considers empirical evidence in corporate finance. Section 3 considers household investments in human capital. Finally, Section 4 considers a more complex setting that illustrates the complementary roles the alternative causal effect estimators can play in as a government seeks to set optimal policies.

\section{Causal Effects in Corporate Finance}

We begin first with a stark example from the field of corporate finance - a field in which quasiexperimental papers have largely displaced the workhorse event-study methodology. Before beginning, we note that a primary objective of empirical corporate finance work is to provide normative advice to CFOs regarding optimal investment and financial policies. With this goal in mind, we turn next to an examination of the role alternative causal effect estimates can play in this domain.

Consider then a CFO facing pressure from a set of investors to sharply increase his corporation's green investments. He is not unsympathetic to their position, with the firm's internal estimates being that the return to green projects is two dollars for each dollar invested. On the other hand, some board members are skeptical.

For simplicity, consider an equity-financed firm with current shares outstanding normalized at 
$1 .^{2}$ Letting $s$ denote shares issued and $p$ the stock price, the corporation's green investment will be:

$$
i(s) \equiv s p(s)
$$

The CFO's objective is to maximize the value of the claim held by current shareholders, subject to the constraint that he no more than double shares outstanding, since this would risk transferring corporate control. Letting $\alpha$ denote the value of assets in place, the CFO's program can be written as:

$$
\max _{0 \leq s \leq 1} \frac{\alpha+2 i(s)}{1+s}=\frac{\alpha+2 s p(s)}{1+s}
$$

Unfortunately, the CEO does not know the price function $p(\cdot)$. Fortunately, last year the government implemented a green investment experiment intended to provide scientific evidence on the returns to such projects. In particular, the government randomly determined the number of shares that a set of comparable firms were to issue, mandating that all proceeds raised be used to fund green investments.

The results of the government's green natural experiment, as studied in a high-profile paper, are represented by the upward sloping line in Figure 1. As shown, stock prices were higher for companies that were randomly-assigned to issue more shares. In fact, the empirically-observed price reaction function has been estimated as

$$
\widetilde{p}(s)=\frac{1}{1-s} .
$$

When pushed by a theorist on the estimated price function, the econometrician studying the experiment provided the following justification. The average value of $\alpha$, denoted $\mu_{\alpha}$, has been estimated at 1 . Therefore, if each dollar invested yields a payoff of two dollars, the price function should satisfy

$$
\widetilde{p}(s)=\frac{1+2 s \widetilde{p}(s)}{1+s} \Rightarrow \widetilde{p}(s)=\frac{\mu_{\alpha}}{1-s} .
$$

Based upon the increasing price function, the researcher had concluded, "You can do well while doing good, and the science shows this."

\footnotetext{
${ }^{2}$ Alternatively, assume the firm's debt covenant prohibits additional debt.
} 
Based upon the paper, the CEO plans to issue the maximum number of shares possible since, in his estimation, this will deliver an unbounded payoff to current shareholders, with

$$
\lim _{s \uparrow 1} \frac{\alpha+2 s \tilde{p}(s)}{1+s}=\infty .
$$

Unfortunately, the CEO has fallen into the logical fallacy of assuming that a causal effect (of share issuance on stock price) that obtains under forced random assignment will remain valid when he exercises discretion. Of course, with private information, this is not generally the case.

To illustrate, we now pin down a securities market equilibrium in the spirit of Myers and Majluf (1984) and Krasker (1986), albeit with somewhat different technological assumptions and a focus on empirical implications. Suppose that $\alpha$, the value of assets-in-place, is private information to the CFO. In particular, assume $\alpha$ is uniformly distributed on $[0,2]$. Indeed, under forced random assignment of equity issuance, $s$ contains no signal value regarding $\alpha$ and so the price function $\widetilde{p}$ estimated in the natural experiment (equations (3) and (4)) was indeed correct for that specific environment. However, with discretionary decision-making under private information, the equilibrium price function will change. To see this, note that the first-order condition for the CFO's program (2) is:

$$
2 i^{\prime}(s)=(1+s)^{-1}[\alpha+2 i(s)] .
$$

Conjecturing an LCSE in which the amount of stock issued fully reveals $\alpha$, market equilibrium entails new equity investors provide funding just equal to their expected payoff, or

$$
i(s)=s(1+s)^{-1}[\alpha+2 i(s)] .
$$

Substituting the equity market equilibrium condition into the CFO's first-order condition we obtain the following differential equation

$$
2 s i^{\prime}(s)=i(s) \Rightarrow i(s)=A \sqrt{s} .
$$

where $A$ is a constant to be determined.

In the LCSE the worst type, with $\alpha=0$, should implement their perfect information allocation, issuing the maximum amount of equity $(s=1)$ and funding the maximum technologically feasible 
amount of green investment, call it $i^{\max }$, a constant which can be assumed to be arbitrarily large. We then have

$$
i^{\max }=A \sqrt{1} \Rightarrow i(s)=i^{\max } \sqrt{s} \Rightarrow p(s)=\frac{i(s)}{s}=\frac{i^{\max }}{\sqrt{s}} .
$$

Empirical evidence from a standard corporate finance event study (a sample of observations of equation (9)) is represented by the downward sloping line in Figure 1. Notice, the partial causal effect of share issuance on stock price is positive but the total causal effect is negative. To understand this, note that equations (4) and (7) allow us to write:

$$
p(s)=\widetilde{p}(s)+\frac{\widehat{\alpha}(s)-\mu_{\alpha}}{1-s} \Rightarrow p^{\prime}(s)=\widetilde{p}(s)+\frac{d}{d s} \frac{\widehat{\alpha}(s)-\mu_{\alpha}}{1-s} .
$$

The implication is that the total causal effect $p^{\prime}$ is equal to the positive partial causal effect $\widetilde{p}^{\prime}$ plus a negative signaling effect which apparently dominates here. Intuitively, with random assignment, there is no negative signal conveyed with changes in $s$, so the stock price is increasing in $s$ due to the positive NPV of incremental investments. However, with endogenous discretionary policies, the type our CFO will actually be choosing, the stock price falls with increases in stock issuance since, as shown below, the market will revise down its inference $(\widehat{\alpha})$ about the value of assets-in-place with higher stock issuance.

With the equilibrium pricing schedule under discretionary CFO stock issuances $(p)$ in mind, the correct first-order condition (6) for pinning down the optimal discretionary choice of $s$ is

$$
i^{\max } s^{-1 / 2}=(1+s)^{-1}\left[\alpha+2 i^{\max } s^{1 / 2}\right] .
$$

From the preceding equation it follows that companies with more valuable assets-in-place issue less new shares. In particular: ${ }^{3}$

$$
s^{*}(\alpha)=\left[-\frac{\alpha}{2 i^{\max }}+\frac{1}{2} \sqrt{\left(\frac{\alpha}{i^{\max }}\right)^{2}+4}\right]^{2} .
$$

Thus, the experimentally-based recommendation that the optimum for all firm entails maximum feasible share issuance $\left(s^{*}=1\right)$ would be incorrect for all firms except the lowest type with $\alpha=0$.

\footnotetext{
${ }^{3}$ Let $\Omega=s^{1 / 2}$ and solve the quadratic in $\Omega$, then compute $s=\Omega^{2}$.
} 
The point of this example is more general. While studies that somehow approximate random assignment of corporate policies related to financing, investment, mergers, board composition, etc. may be of some independent scientific interest, they are also likely to be grossly misleading from the perspective of real-world CFOs making endogenous discretionary decisions in capital markets rife with asymmetric information.

\section{Returns on Educational Investments}

This section considers the returns on educational investments. The first subsection considers the causal effect of an elite university education. The second subsection considers the causal effect of incremental education.

\subsection{Causal Effect of Elite Universities}

Suppose all high school graduates within the state that go on to college attend State University ("State U") or Ivy University ("Ivy U"). State U charges a nominal tuition of $\$ 5,000$ per year, or $\$ 20,000$ for a four year degree. Ivy U carries a considerably higher price tag, with tuition amounting to $\$ 50,000$ per year, or $\$ 200,000$ for a four year degree. Given the ten-fold tuition increase, a natural question has arisen: "Is the $\$ 180,000$ added expense of an elite Ivy U education really worth it?"

Conveniently, applied econometricians have recently focused their attention on precisely this question. In particular, Figure 2 plots hypothetical probability distributions of average (capitalized) salary differences, Ivy U minus State U, from two competing studies relying upon two distinct graduating classes, 2018 versus 2019. The 2018 college graduates come from the high school graduating class of 2014. This high school class is typical in the sense that, at the time of college entry, the students were free to choose between colleges, as is the norm within the U.S. The 2019 college graduates come from the high school graduating class of 2015. This high school class was very special in that the government, in the interest of science, compelled graduates to participate in a scheme whereby they were forcibly randomly assigned to State U or Ivy U. 
As shown in Figure 2, the causal conclusions of the two study types tend to differ dramatically. In particular, the average salary difference in present value terms is $\$ 422,000$ under random assignment, but is $\$ 1,155,000$ for the cohort not subjected to randomization. With these figures in mind, it is worthwhile for the reader to consider a fundamental question: Which causal effect estimate is of greater general interest?

We venture to argue that the answer to the prior question is not clear. Before elaborating on this, consider the data generating process. Institutions competitively supply educational intensities $e \in[0, \infty)$ at a per student cost $\phi e .^{4}$ Prospective students have educational effort cost functions $e^{2} / \theta$, where the latent ability parameter $\theta \in\{\underline{\theta}, \bar{\theta}\}$, with $0<\underline{\theta}<\bar{\theta}$. Each type occurs with positive probability and the expected value of $\theta$ is denoted $\mu_{\theta}$. There is a competitive labor market where firms compete á la Bertrand and will offer an employee their expected productivity given the firms' common beliefs. The productivity of a type $\theta$ college graduate is

$$
\theta(1+m e)
$$

where $m>\phi / \underline{\theta}$, which implies even low types would get some education under perfect information. Given discretion, prospective students play pure strategies and maximize their net utility $u$ which is the difference between their anticipated wage and the total costs of education, tuition plus effort costs. If any two universities pool at a common educational intensity $e$, only then will students mix, attending each with, say, equal probability.

Consider then a perfect Bayesian equilibrium (PBE) in which all students attend either Ivy $\mathrm{U}$ or State U, which are configured to offer respective educational intensities $\{\underline{e}, \bar{e}\}$. To begin, note any PBE sustainable is sustainable by imputing to type $\underline{\theta}$ any $e \notin\{\underline{e}, \bar{e}\}$. Next, define the following type-contingent maximal payoff given worst-case beliefs:

$$
u_{\theta}^{D} \equiv \max _{e \geq 0} \underline{\theta}(1+m e)-\phi e-\frac{e^{2}}{\theta}=\underline{\theta}+\frac{1}{4} \theta(m \underline{\theta}-\phi)^{2} .
$$

\footnotetext{
${ }^{4}$ See Hara, Segal and Tadelis (1997) without tuition. However, their equilibrium characterization is incorrect. Setting $\phi=0$ in our lemma corrects the error.
} 
In any separating equilibrium, low types get their full-information payoff $u_{\underline{\theta}}^{D}$ since lower payoffs would induce deviations and higher payoffs are inconsistent with competitive wage-setting. At the minimal Ivy U educational intensity in a separating equilibrium, low types are just indifferent to deviating, leaving high types strictly preferring not deviating. At the maximal Ivy U educational intensity in a separating equilibrium, high types derive net utility $u \frac{D}{\theta}$ and are just indifferent to deviating, leaving low types strictly preferring not deviating. At the maximum educational intensity amongst pooling equilibria, low types are just indifferent to deviating and high types strictly prefer not deviating. Figure 3 illustrates these potential equilibrium configurations in tuition-salary space. The indifference curves pin each type to their respective deviation utilities $u_{\theta}^{D}$.

From the preceding indifference conditions, we have the following lemma.

LEMMA 1. In all separating equilibria, State University caters to low ability students, setting $\underline{e}^{*}=\underline{\theta}(m \underline{\theta}-\phi) / 2$ and charging tuition $\phi \underline{e}^{*}$. Ivy University caters to high ability students, charging higher tuition $\phi \bar{e}^{*}$, where

$$
\bar{e}^{*} \in\left[\frac{\underline{\theta}(m \bar{\theta}-\phi)+\sqrt{\underline{\theta}^{2}(m \bar{\theta}-\phi)^{2}+4 \underline{\theta}\left(\bar{\theta}-u_{\underline{\theta}}^{D}\right)}}{2}, \frac{\bar{\theta}(m \bar{\theta}-\phi)+\sqrt{\bar{\theta}^{2}(m \bar{\theta}-\phi)^{2}+4 \bar{\theta}\left(\bar{\theta}-u_{\bar{\theta}}^{D}\right)}}{2}\right] .
$$

The universities can instead pool charging a common tuition $\phi e^{* *}$ where $e^{* *}$ is not greater than

$$
e^{* *}=\frac{1}{2}\left[\underline{\theta}\left(m \mu_{\theta}-\phi\right)+\sqrt{\underline{\theta}^{2}\left(m \mu_{\theta}-\phi\right)^{2}+4 \underline{\theta}\left(\mu_{\theta}-u_{\underline{\theta}}^{D}\right)}\right] .
$$

With these potential equilibria in mind, consider again the question of the general interest of the two competing studies. For starters, note that random assignment provides no additional benefit if the universities are configured as in any of the pooling equilibria described in Lemma 1. After all, in the event of pooling, employers will offer wage $\left(1+m e^{* *}\right) \mu_{\theta}$ to both Ivy U and State $\mathrm{U}$ graduates, regardless of whether or not random assignment has taken place. Therefore, in the event of pooling, studies with and without random assignment would generally find zero salary differential, consistent with the reality of zero causal effect. 
Suppose instead that the two universities implement one of the separating equilibria described in Lemma 1. This is precisely the situation depicted in Figure 2, which plotted the theoretical distribution of the salary differentials for studies with and without random assignment, assuming the least-cost separating equilibrium (LCSE) is implemented, with i.i.d. normally distributed salary measurement errors. The figure assumes an educational productivity parameter $m=.03$, with $\underline{\theta}=$ 56.5 and $\bar{\theta}=155$, with $10 \%$ being high types. These parameters were chosen to generate a realistic tuition differential, with Ivy charging tuition of $\$ 200,000$ and State U charging tuition of $\$ 20,000$, as captured in the LCSE in Figure 3.

One could readily argue that the study without random assignment is of greater general interest. After all, perhaps the most important consumers of such studies are the millions of prospective college students who stand to make life-altering decisions based upon them. For the vast majority of them, the relevant question is: Does the (present value) of the salary increase provided by attending Ivy U exceed the incremental costs? For this purpose, the more relevant study would be that relying on the 2018 cohort where students were, as usual, free to choose and free to signal, with signal content reflected in wages.

This argument can be expressed more formally. Consider that, if the universities are configured as in any of the separating equilibria, the respective wage differential, or causal effects, in studies with and without random assignment are:

$$
\begin{aligned}
\bar{w}^{R}-\underline{w}^{R} & =\left(1+m \bar{e}^{*}\right) \mu_{\theta}-\left(1+m \underline{e}^{*}\right) \mu_{\theta}=m \mu_{\theta}\left(\bar{e}^{*}-\underline{e}^{*}\right) . \\
\bar{w}^{*}-\underline{w}^{*} & =\bar{\theta}\left(1+m \bar{e}^{*}\right)-\underline{\theta}\left(1+m \underline{e}^{*}\right)=\left(1+m \underline{e}^{*}\right)(\bar{\theta}-\underline{\theta})+m \bar{\theta}\left(\bar{e}^{*}-\underline{e}^{*}\right) .
\end{aligned}
$$

If we then take the perspective of a prospective college student, participating in a normal education/labor market, the notion of causal effect implicit under random assignment carries two sources of error. First, it fails to reflect the ability signaling value, the first term in the final $\bar{w}^{*}-\underline{w}^{*}$ expression above. Second, the causal productivity effect derived under random assignment reflects average ability. However, selecting the elite university causes the labor market to infer high ability, and this amplifies the productivity effect. In fact, the preceding equation allows us to rewrite the 
total causal effect as the partial causal effect plus terms capturing the increase in payoff arising from imputation of high-type status:

$$
\bar{w}^{*}-\underline{w}^{*}=\bar{w}^{R}-\underline{w}^{R}+\left(1+m \underline{e}^{*}\right)(\bar{\theta}-\underline{\theta})+m\left(\bar{\theta}-\mu_{\theta}\right)\left(\bar{e}^{*}-\underline{e}^{*}\right) .
$$

Returning to a student's college decision, relying on the random assignment study would lead high ability types to incorrectly attend State U. To see this, first note that high ability students are strictly better off in the LCSE than under their next best alternative (not State U), with the net utility gain being

$$
\bar{\theta}\left(1+m \bar{e}^{*}\right)-\phi \bar{e}^{*}-\frac{\left(\bar{e}^{*}\right)^{2}}{\theta}-u_{\bar{\theta}}^{D}=\$ 609,000 .
$$

However, if a high ability type were to rely on the random assignment study they would incorrectly opt for State U, calculating a negative Ivy U net utility gain:

$$
\bar{w}^{R}-\underline{w}^{R}-\phi\left(\bar{e}^{*}-\underline{e}^{*}\right)-\frac{\left(\bar{e}^{*}\right)^{2}-\left(\underline{e}^{*}\right)^{2}}{\bar{\theta}}=-\$ 113,000 .
$$

Having said all this, other potential readers will be more interested in, say, testing the null hypothesis that elite universities serve no productive purpose $(m=0)$. To test that null hypothesis, clearly the study with random assignment would be superior. After all, without random assignment, the very signaling value of interest to prospective students serves to cloud measurement of the pure productivity effect (on the treated). Nevertheless, the point remains that for some decision-makers and margins, knowledge of the signal value of actions is necessary to choose optimal actions, and random assignment shuts this down, by construction.

\subsection{Causal Effect of Additional Education}

The issues discussed above can be further refined by considering a slight variation on the setting. In particular, suppose that instead of there being only two types, suppose $\theta \in[0, \infty) .{ }^{5}$ And further, suppose for simplicity that education serves no productive purpose, with $m=0$. Finally, consider

\footnotetext{
${ }^{5}$ See Hara, Segal and Tadelis (1997) without tuition.
} 
once again the LCSE. In this case, agents solve:

$$
\max _{\widetilde{e}} w(\widetilde{e})-\widetilde{e}^{2} / \theta-\phi \widetilde{e} \Rightarrow w^{\prime}(e)-2 e / \theta-\phi=0 .
$$

Competitive wage setting implies $w(e)=\theta$. Combining the wage equation with the first-order condition we obtain the following ODE:

$$
w(e) w^{\prime}(e)-\phi w(e)-2 e=0 .
$$

We conjecture a solution taking the linear form $w(e)=\kappa e$ for some constant $\kappa>0$. Under this conjecture, $w(0)=0$, consistent with the lowest $\theta=0$ types getting zero education, as under perfect information. Substituting the conjecture into the ODE and solving for $\kappa$ we find the following LCSE wage schedule:

$$
w^{*}(e)=\frac{e}{\sqrt{2}} \sqrt{\left(\phi^{2}+4\right)+\phi \sqrt{\phi^{2}+8}} \Rightarrow \frac{d w^{*}(e)}{d e}=\frac{1}{\sqrt{2}} \sqrt{\left(\phi^{2}+4\right)+\phi \sqrt{\phi^{2}+8}} .
$$

With the preceding result in mind, consider the interpretation and utilization of microeconometric evidence concerning whether the returns on the quantity/quality of education $(e)$ justify the extra tuition $\phi e$. To fix ideas, one might well envision econometricians finding this topic to be of particular importance if they observe an increase in tuitions, driven, say, by an increase in the costs of producing education $\phi$. In the present setting, a study randomly assigning a given cohort across universities would conclude that education is a terrible investment, since under randomization, all graduates from the randomized cohort would be paid $\mu_{\theta}$, with an implied salary causal effect of 0 for any education increment $\Delta e$, as compared with an incremental tuition increase $\phi \Delta e$. This situation is depicted by the thick solid education-wage schedule in Figure 4.

However, as shown by in the preceding equation, the true equilibrium salary increment will naturally increase with the marginal cost of producing education $\phi$ so as to restore signaling incentives. Figure 4 illustrates. Notice, the equilibrium wage profile naturally becomes steeper with increases in the marginal cost $\phi$ of producing higher education. By construction, randomization shuts off this channel. Neglect of this fact can then lead to faulty cost-benefit assessments in higher education, especially as costs of education production increase over time. 


\section{Evidence-Based Government Policy}

Much of the motivation given for random assignment is based upon the notion that the resulting causal estimates are particularly valuable in terms of setting optimal government policies. With this in mind, this section considers a more detailed and complex example of how the two forms of causal effects, partial and total causal effects, can both be used by a government policymaker in a complementary fashion. The government's decision problem is a common one, how to use empirical evidence to decide on regulatory and tax policies.

Time is continuous and horizons are infinite. Agents are risk-neutral and discount cash flows at the risk-free rate $r$. Firms (or other agents) accumulate a stock (say capital) according to the law of motion

$$
d K=(I-\delta K) d t
$$

with the price of a unit of capital being 1 and adjustment costs being $\gamma I^{2}$.

A government (e.g. state or national) has discretion to choose the state of its policy variable. We will call this economy, the "endogenous policy economy." The policy state is binary, $S \in\{S 1, S 2\}$. The policy variable influences marginal product, and with it, investment. In particular, in policy state $S$ the marginal product is $\Pi_{S} X K$, where $X$ is a geometric Brownian motion evolving according to

$$
\frac{d X_{t}}{X_{t}}=\mu_{t} d t+\sigma d W
$$

where $W$ is a standard Wiener process. We shall think of $X$ as representing an aggregate shock hitting firms in the endogenous policy economy. Notice, the drift $\mu_{t}$ is time-varying. In particular, as described in greater detail below, we assume $\mu_{t}$ is a binary stochastic process. The realization of this process is private information to the government.

For the sake of the illustration, assume

$$
\Pi_{S 2}>\Pi_{S 1}
$$

The first causal inference problem is that, by assumption, the government does not know the pre- 
ceding inequality, nor the magnitude of either $\Pi_{S}$. That is, the government does not know which policy variable state is technologically more stimulative.

Suppose now that there is a neighboring economy (the "experimental policy economy") identical in all respects to the endogenous policy economy, but with the exception that this neighbor randomizes its policy variable, alternating between $\Pi_{S 1}$ and $\Pi_{S 2}$. In particular, over any infinitesimal time interval $d t$, with probability $\lambda d t$ the policy variable will switch states. This stochastic process is independent of any other random variable including the aggregate shock hitting the experimental economy which has the following law of motion

$$
\frac{d \widetilde{X}}{\widetilde{X}}=\widetilde{\mu}_{t} d t+\sigma d \widetilde{W}
$$

where $\widetilde{W}$ is also a standard Wiener process and $\widetilde{\mu}_{t}$ is also a binary stochastic process that is unobservable to firms, with identical probability law as $\mu_{t}$. The fact that the experimental policy economy is endowed with the same probability law for the aggregate shock as the endogenous policy economy makes it a convenient benchmark.

The government of the endogenous policy economy will first attempt to use evidence from the experimental policy economy's shock responses to infer the (relative) magnitudes of $\Pi_{S 1}$ and $\Pi_{S 2}$. Since the neighbor randomizes its policy variable, this first step will be an exercise in estimating partial causal effects.

Assuming the optimizing government is successful in determining which policy state is more stimulative in terms of underlying latent technological parameters, it faces a second challenge: determining the magnitude of total causal effects. In particular, we assume the government in the endogenous policy economy will adopt as a policy rule switching to the more (less) stimulative policy if the current instantaneous aggregate drift is low (high). However, as we show, since the aggregate drift is private information to the government, the response to policy variable changes will be dampened (and potentially reversed) due to the opposing signal content. Here the econometrician must estimate total causal effects in order to correctly predict how firms will respond to discretionary policy interventions. 


\subsection{Experimental Policy}

We consider first the experimental policy economy in which the policy variable is an exogenous stochastic process. Following Veronesi (2000), assume that the instantaneous drift can take on two potential values, $\mu_{1}>\mu_{2}$. This holds true in both economies.

Recall, the drift is unobservable to all parties except the government. Over any infinitesimal time interval $d t$ with probability $p d t$ a drift will be randomly drawn according to the probability distribution $\mathbf{f}=\left(f_{1}, f_{2}\right)$. Let $\mathbf{Z}$ be the two-dimensional vector of probability weights agents place on each potential drift and let

$$
\mu(\mathbf{Z}) \equiv Z_{1} \mu_{1}+Z_{2} \mu_{2}
$$

In the experimental economy, the government provides no signals, so agents must instead form inferences based upon the realized path of $\tilde{X}$. From Lemma 1 in Veronesi (2000) it follows beliefs evolve as diffusions, with:

$$
d Z_{n}=\underbrace{p\left(f_{n}-Z_{n}\right)}_{\equiv \mu_{z_{n}}} d t+\underbrace{\frac{Z_{n}\left[\mu_{n}-\mu(\mathbf{Z})\right]}{\sigma}}_{\equiv \sigma_{z_{n}}} \widetilde{W} .
$$

The Hamilton-Jacobi-Bellman (HJB) equation for the firm is:

$$
\begin{aligned}
& r V(K, X, S, \mathbf{Z}) \\
= & \max _{I} \Pi_{S} K X-I-\gamma I^{2}+V_{k}(I-\delta K)+V_{x} \mu(\mathbf{Z}) X+\mu_{z_{1}} V_{z_{1}}+\mu_{z_{2}} V_{z_{2}} \\
& +\lambda\left[V\left(K, X, S^{\prime}, \mathbf{Z}\right]-V(K, X, S, \mathbf{Z}]+\frac{1}{2} V_{x x} \sigma^{2} X^{2}+\frac{1}{2} V_{z_{1} z_{1}} \sigma_{z_{1}}^{2}+\frac{1}{2} V_{z_{2} z_{2}} \sigma_{z_{2}}^{2}\right. \\
& +V_{z_{1} z_{2}} \sigma_{z_{1}} \sigma_{z_{2}}+V_{x z_{1}} X \sigma \sigma_{z_{1}}+V_{x z_{2}} X \sigma \sigma_{z_{2}} .
\end{aligned}
$$

The HJB equation can be understood as an equilibrium condition demanding that the expected holding return on the firm's stock must be just equal to the opportunity cost. The holding return consists of dividends plus expected capital gains. In turn, the capital gains can be understood as a second-order Taylor expansion using the rules of Ito calculus.

We conjecture and verify that the value function takes the following separable form:

$$
r V(K, X, S, \mathbf{Z})=K Q(X, S, \mathbf{Z})+G(X, S, \mathbf{Z})
$$


Isolating those terms in the HJB equation involving the instantaneous investment control we find that the optimal investment policy solves

$$
\max _{I} I Q(X, S, \mathbf{Z})-I-\gamma I^{2} \Rightarrow I^{*}(X, S, \mathbf{Z})=\frac{Q(X, S, \mathbf{Z})-1}{2 \gamma} .
$$

That is, investment is linear in the shadow value of capital $Q$.

Next we note that since the HJB equation must hold pointwise, the terms scaled by $K$ must equate. Using this fact, we obtain an equation for pinning down the shadow value of capital $Q$ :

$$
\begin{aligned}
& (r+\delta+\lambda) Q(X, S, \mathbf{Z}) \\
= & \Pi_{S} X+\mu(\mathbf{Z}) X Q_{x}+\mu_{z_{1}} Q_{z_{1}}+\mu_{z_{2}} Q_{z_{2}}+\lambda Q\left(X, S^{\prime}, \mathbf{Z}\right) \\
& +\frac{1}{2} \sigma^{2} X^{2} Q_{x x}+\frac{1}{2} \sigma_{z_{1}}^{2} Q_{z_{1} z_{1}}+\frac{1}{2} \sigma_{z_{2}}^{2} Q_{z_{2} z_{2}} \\
& +\sigma_{z_{1}} \sigma_{z_{2}} Q_{z_{1} z_{2}}+X \sigma \sigma_{z_{1}} Q_{x z_{1}}+X \sigma \sigma_{z_{2}} Q_{x z_{2}}
\end{aligned}
$$

Now let $X \Psi_{S}^{n}$ denote the shadow value of capital in policy state $S$ given drift rate $\mu_{n}$. As shown in the Online Appendix, we have the following lemma pinning down the solution to the preceding shadow value equation for the experimental economy.

LEMMA 2. In the experimental economy, the shadow value of capital is

$$
Q(X, S, \mathbf{Z})=X\left[Z_{1} \Psi_{S}^{1}+Z_{2} \Psi_{S}^{2}\right]
$$

where the shadow value constants solve the following linear system

$$
\begin{aligned}
& {\left[r+\delta-\mu_{1}+\lambda+p f_{2}\right] \Psi_{S 1}^{1}=\Pi_{S 1}+p f_{2} \Psi_{S 1}^{2}+\lambda \Psi_{S 2}^{1}} \\
& {\left[r+\delta-\mu_{2}+\lambda+p f_{1}\right] \Psi_{S 1}^{2}=\Pi_{S 1}+p f_{1} \Psi_{S 1}^{1}+\lambda \Psi_{S 2}^{2}} \\
& {\left[r+\delta-\mu_{1}+\lambda+p f_{2}\right] \Psi_{S 2}^{1}=\Pi_{S 2}+p f_{2} \Psi_{S 2}^{2}+\lambda \Psi_{S 1}^{1}} \\
& {\left[r+\delta-\mu_{2}+\lambda+p f_{1}\right] \Psi_{S 2}^{2}=\Pi_{S 2}+p f_{1} \Psi_{S 2}^{1}+\lambda \Psi_{S 1}^{2}}
\end{aligned}
$$


Subtracting the first equation listed in the lemma from the third and the second equation from the fourth, the following inequalities are readily verified:

$$
\Pi_{S 2}>\Pi_{S 1} \Rightarrow \Psi_{S 2}^{1}>\Psi_{S 1}^{1}, \Psi_{S 2}^{2}>\Psi_{S 1}^{2}
$$

We then have the following important proposition showing the utility of natural policy experiments in determining the relative magnitude of technological parameters. Of course, inferring technological parameters is often a natural pre-requisite for setting policy optimally, and this is the case here.

PROPOSITION 1. Observing any shock response in the experimental economy allows for a correct ranking of partial causal effects, with the response to an exogenous transition from $S$ to $S^{\prime}$ being

$$
S R_{S S^{\prime}}=\frac{X}{2 \gamma} \times\left[Z_{1}\left(\Psi_{S^{\prime}}^{1}-\Psi_{S}^{1}\right)+Z_{2}\left(\Psi_{S^{\prime}}^{2}-\Psi_{S}^{2}\right)\right]
$$

Notwithstanding the positive conclusion of the proposition, that natural experiments here allow for a correct ranking of relative stimulus provided by the alternative policies (sans-signaling), it is also clear that latent time-varying beliefs $(\mathbf{Z})$ will make it hard for the government to correctly infer the absolute magnitudes of the technological parameters. Anticipating, this will be problematic since, once policy discretion is introduced, there will be a signaling effect working in the opposite direction of the partial causal effect.

\subsection{Endogenous Policy}

Suppose now that, based upon the experimental evidence (Proposition 1), the government of the endogenous policy economy tries to lean against the wind, implementing policy $\Pi_{S 2}$ whenever it privately observes that the drift rate is low $\left(\mu_{t}=\mu_{2}\right)$ and $\Pi_{S 1}$ whenever it privately observes that the drift rate is high $\left(\mu_{t}=\mu_{1}\right)$, recalling $\mu_{1}>\mu_{2}$. What will be the observed total causal effect?

The HJB equation for the firm here is:

$$
\begin{aligned}
& r V(K, X, S) \\
= & \max _{I} \Pi_{S} K X-I-\gamma I^{2}+V_{k}(I-\delta K)+V_{x} \mu_{S} X+ \\
& \left.+p\left(1-f_{S}\right)\left[V\left(K, X, S^{\prime}\right)\right]-V(K, X, S)\right]+\frac{1}{2} V_{x x} \sigma^{2} X^{2} .
\end{aligned}
$$


We conjecture and verify that the value function takes the following separable form:

$$
V(K, X, S)=K q(X, S)+g(X, S)
$$

Isolating those terms in the HJB equation involving the instantaneous investment control we find that the optimal investment policy solves

$$
\max _{I} I q(X, S)-I-\gamma I^{2} \Rightarrow I^{*}(X, S)=\frac{q(X, S)-1}{2 \gamma} .
$$

Next we note that since the HJB equation must hold pointwise, the terms scaled by $K$ must equate. Using this fact and rearranging terms we obtain an equation for pinning down the shadow value of capital:

$$
\left[r+\delta+p\left(1-f_{S}\right)\right] q(X, S)=\Pi_{S} X+\mu_{S} X q_{x}(X, S)+\frac{1}{2} \sigma^{2} X^{2} q_{x x}(X, S)+p\left(1-f_{S}\right) q\left(X, S^{\prime}\right)
$$

Since the dividend is linear in $X$ we conjecture the shadow value is also linear in $X$ taking the form

$$
q(X, S)=X \psi_{S}
$$

Substituting this into the preceding equation and rearranging terms we obtain the following two equations pinning down the shadow values in the endogenous policy economy

$$
\begin{aligned}
& {\left[r+\delta-\mu_{1}+p\left(1-f_{1}\right)\right] \psi_{1}=\Pi_{S 1}+p\left(1-f_{1}\right) \psi_{2}} \\
& {\left[r+\delta-\mu_{2}+p\left(1-f_{2}\right)\right] \psi_{2}=\Pi_{S 2}+p\left(1-f_{2}\right) \psi_{1} .}
\end{aligned}
$$

Solving this system we find

$$
\begin{aligned}
\psi_{1}= & \frac{\Pi_{S 1}}{r+\delta-\mu_{1}} \\
& +\frac{p\left(1-f_{1}\right)\left(r+\delta-\mu_{2}\right)\left[\Pi_{S 2} /\left(r+\delta-\mu_{2}\right)-\Pi_{S 1} /\left(r+\delta-\mu_{1}\right)\right]}{\left(r+\delta-\mu_{1}\right)\left(r+\delta-\mu_{2}\right)\left[1+p\left(1-f_{1}\right) /\left(r+\delta-\mu_{1}\right)+p\left(1-f_{2}\right) /\left(r+\delta-\mu_{2}\right)\right]}
\end{aligned}
$$

with the symmetric expression for $\psi_{2}$.

Since investment is increasing in $q \equiv X \psi_{S}$, implementation of $\Pi_{S 2}$ will be followed by an increase in investment iff $\psi_{2}>\psi_{1}$. Comparing the shadow value constants from equation (38) we have the following proposition. 
PROPOSITION 2. Investment will increase after discretionary government implementation of the technologically stimulative policy $\Pi_{S 2}>\Pi_{S 1}$ if and only if the partial causal effect is sufficiently large to imply

$$
\frac{\Pi_{S 2}}{\Pi_{S 1}}>\frac{r+\delta-\mu_{2}}{r+\delta-\mu_{1}}
$$

Essentially, the preceding proposition shows that the total causal effect will have the same sign as the partial causal effect if and only if the latter causal effect is sufficiently large to offset the negative signal regarding aggregate drift. To see this, note that the condition in the proposition can be stated in terms of shadow values under constant policies and drifts:

$$
\frac{\Pi_{S 2}}{r+\delta-\mu_{2}}>\frac{\Pi_{S 1}}{r+\delta-\mu_{1}} \Rightarrow q(X, S 2)>q(X, S 1) .
$$

The point of this example is to illustrate in a concrete way how both forms of causal effect estimates can play a critical role in policy-setting. Perhaps ironically, here partial causal effects were shown to be especially helpful in terms of inferring deep technological parameters. But once these technological parameters are assessed, it seems that (perhaps) only through actually implementing the policy rule in a discretionary fashion can the government get a better sense of how things will work in reality. Moreover, in contrast to the present stylized example, in reality it will be hard to predict the magnitude of signaling effects since the true nature and quality of agent information is hard for a government to know with a high degree of precision. In fact, in reality, information quality and beliefs will vary over time, giving rise to time-varying signal content in many applied settings. This might well be an interesting direction for future applied theory and empirical work.

\section{Conclusion}

This paper questions the notion that evidence from random assignment is somehow more "credible" than more traditional forms of evidence in finance such as event studies. As we show, if a prospective decision-maker is privately informed and is indeed attempting to make optimal discretionary decisions, rather than attempting to understand the consequences of random mistakes, then 
the signal content of her decisions is payoff-relevant and decision-relevant. Causal effect estimates derived from random assignment strip out signaling effects, potentially leaving the decision-maker ignorant of the true implications of alternative decisions. Nevertheless, we show how partial causal effect estimates can be used in conjunction with total causal effect estimates to decompose real and information signaling channels. As shown, in practice, both forms of empirical estimates, partial and total causal effects, may be necessary inputs in order to pin down optimal policies. If this fact is appreciated, then a broader definition of "identification" should be adopted, and a broader set of evidence should be treated as "credible." 


\section{References}

[1] Alti, Aydogan, 2003, How Sensitive is Investment to Cash Flow when Financing is Frictionless? The Journal of Finance 58, 707-722.

[2] Angrist, Joshua D. and Jorn-Steffen Pischke, 2009, Mostly Harmless Econometrics: An Empiricist's Companion, Princeton University Press.

[3] Angrist, Joshua D. and Jorn-Steffen Pischke, 2015, Mastering Metrics: The Path from Cause to Effect, Princeton University Press.

[4] Athreya, Kartik and Janice Eberly, 2020, Risk, the College Premium, and Aggregate Human Capital Investment, forthcoming in American Economic Journal: Macroeconomics.

[5] Becker, Gary, 1993, Human Capital: A Theoretical and Empirical Analysis, with Specific Reference to Education, University of Chicago Press.

[6] Bowen, Donald E., Laurent Fresard, and Jerome Taillard, 2016, What's Your Identification Strategy? Innovation in Corporate Finance Research, Management Science, 2529-2548.

[7] Chemla, Gilles and Christopher A. Hennessy, 2019, Controls, Belief Updating, and Bias in Medical RCTs, Journal of Economic Theory.

[8] Duflo, Esther, 2004, Scaling Up and Evaluation, in Annual World Bank Conference on Development Economics: Accelerating Development, ed. François Bourguignon and Boris Pleskovic, 341-69. Washington, D.C.: World Bank; Oxford and New York: Oxford University Press.

[9] Fama, Eugene, Lawrence Fisher, Michael Jensen, and Richard Roll, 1969, The Adjustment of Stock Prices to New Information, International Economic Review 10, 1-28.

[10] Fudenberg, Drew and David K. Levine, 2020, Learning in Games and the Interpretation of Natural Experiments, forthcoming in American Economic Journal: Microeconomics.

[11] Gomes, Joao, 2001, Financing Investment, American Economic Review 91, 1263-1285. 
[12] Hara, Chiaki, Ilya Segal, and Steve Tadelis, 1997, Solutions Manual for Microeconomic Theory, Oxford University Press.

[13] Imbens, Guido W. and Donald B. Rubin, 2015, Causal Inference for Statistics, Social, and Biomedical Sciences: An Introduction, Cambridge University Press.

[14] Keane, Michael P., Structural vs. Atheoretic Approaches to Econometrics, 2010, Journal of Econometrics 156.

[15] Krasker, William, 1986, Stock Price Movements in Response to Stock Issues under Asymmetric Information, Journal of Finance.

[16] Leland, Hayne and David Pyle, 1977, Information Asymmetry, Financial Structure, and Financial Intermediation, Journal of Finance 32, 371-387.

[17] Löfgren, Karl-Gustaf, Torsten Persson, and Jorgen Weibull, 2002, Markets with Asymmetric Information: The Contributions of George Akerlof, Michael Spence and Joseph Stiglitz, Scandinavian Journal of Economics 104(2), 195-211.

[18] Moyen, Nathalie, 2005, Investment-Cash Flow Sensitivities: Constrained vs Unconstrained Firms. Journal of Finance 59, 2061-2092.

[19] Myers, Stewart, and Nicholas S. Majluf, 1984, Corporate Financing and Investment Decisions when Firms have Information that Investors do not Have, Journal of Financial Economics 13, 187-221.

[20] Philipson, Tomas, and Jeffrey Desimone, 1997, Experiments and Subject Sampling, Biometrika, 619-630.

[21] Riley, John G., 2001, Silver Signals: Twenty-Five Years of Screening and Signaling, Journal of Economic Literature, 432-478. 
[22] Ross, Stephen, 1977, The Determination of Financial Structure: The Incentive Signaling Approach, Bell Journal of Economics, 23-40.

[23] Roussanov, Nikolai, 2010, Human Capital Investment and Portfolio Choice over the Life Cycle, working paper, Wharton Business School.

[24] Rubin, Donald B., 1974, Estimating the Causal Effects of Treatments in Randomized and Non-Randomized Studies, Journal of Educational Psychology 66, 688-701.

[25] Rust, John, 2014, The Limits of Inference with Theory: A Review of Wolpin 2013, Journal of Economic Literature.

[26] Spence, Michael, 1973, Job Market Signaling, Quarterly Journal of Economics 87(3), 355-374.

[27] Veronesi, Pietro, 2000, How Does Information Quality Affect Stock Returns? Journal of Finance, $807-837$. 


\section{Signaling, Random Assignment, and Causal Effect Estimation: Online Appendix}

February 2020

\section{Experimental Economy Shadow Value}

Following Veronesi (2000), assume that the instantaneous drift of aggregate output $\widetilde{X}$ can take on two potential values, $\mu_{1}>\mu_{2}$. Recall, the drift is unobservable to all parties except the government. Over any infinitesimal time interval $d t$ with probability $p d t$ a drift will be randomly drawn according to the probability distribution $\mathbf{f}=\left(f_{1}, f_{2}\right)$. Let $\mathbf{Z}$ be the two-dimensional vector of probability weights agents place on each potential drift and let

$$
\mu(\mathbf{Z}) \equiv Z_{1} \mu_{1}+Z_{2} \mu_{2}
$$

Since here the government provides no signals, agents must instead form inferences based upon the realized path of $\widetilde{X}$.

From Lemma 1 in Veronesi (2000) it follows macroeconomic beliefs evolve as diffusions, with:

$$
d Z_{n}=\underbrace{p\left(f_{n}-Z_{n}\right)}_{\equiv \mu_{z_{n}}} d t+\underbrace{\frac{Z_{n}\left[\mu_{n}-\mu(\mathbf{Z})\right]}{\sigma}}_{\equiv \sigma_{z_{n}}} \widetilde{W} .
$$


The Hamilton-Jacobi-Bellman (HJB) equation for the firm is:

$$
\begin{aligned}
& r V(K, X, S, \mathbf{Z}) \\
= & \max _{I} \Pi_{S} K X-I-\gamma I^{2}+V_{k}(I-\delta K)+V_{x} \mu(\mathbf{Z}) X+\mu_{z_{1}} V_{z_{1}}+\mu_{z_{2}} V_{z_{2}} \\
& +\lambda\left[V\left(K, X, S^{\prime}, \mathbf{Z}\right]-V(K, X, S, \mathbf{Z}]+\frac{1}{2} V_{x x} \sigma^{2} X^{2}+\frac{1}{2} V_{z_{1} z_{1}} \sigma_{z_{1}}^{2}+\frac{1}{2} V_{z_{2} z_{2}} \sigma_{z_{2}}^{2}\right. \\
& +V_{z_{1} z_{2}} \sigma_{z_{1}} \sigma_{z_{2}}+V_{x z_{1}} X \sigma \sigma_{z_{1}}+V_{x z_{2}} X \sigma \sigma_{z_{2}} .
\end{aligned}
$$

The HJB equation can be understood as an equilibrium condition demanding that the expected holding return on the firm's stock must be just equal to the opportunity cost. The holding return consists of dividends plus expected capital gains. In turn, the capital gains can be understood as a second-order Taylor expansion using the rules of Ito calculus.

We conjecture and verify that the value function takes the following separable form:

$$
r V(K, X, S, \mathbf{Z})=K Q(X, S, \mathbf{Z})+G(X, S, \mathbf{Z})
$$

Substituting the conjectured value function into the HJB equation we obtain

$$
\begin{aligned}
& (r+\lambda)[K Q(X, S, \mathbf{Z})+G(X, S, \mathbf{Z})] \\
= & \max _{I} \Pi_{S} K X-I-\gamma I^{2} \\
& +(I-\delta K) Q+\mu(\mathbf{Z}) X\left[K Q_{x}+G_{x}\right]+\mu_{z_{1}}\left[K Q_{z_{1}}+G_{z_{1}}\right]+\mu_{z_{2}}\left[K Q_{z_{2}}+G_{z_{2}}\right] \\
& +\lambda\left[K Q\left(X, S^{\prime}, \mathbf{Z}\right)+G\left(X, S^{\prime}, \mathbf{Z}\right)\right] \\
& +\frac{1}{2} \sigma^{2} X^{2}\left[K Q_{x x}+G_{x x}\right]+\frac{1}{2} \sigma_{z_{1}}^{2}\left[K Q_{z_{1} z_{1}}+G_{z_{1} z_{1}}\right]+\frac{1}{2} \sigma_{z_{2}}^{2}\left[K Q_{z_{2} z_{2}}+G_{z_{2} z_{2}}\right] \\
& +\sigma_{z_{1}} \sigma_{z_{2}}\left[K Q_{z_{1} z_{2}}+G_{z_{1} z_{2}}\right]+X \sigma \sigma_{z_{1}}\left[K Q_{x z_{1}}+G_{x z_{1}}\right]+X \sigma \sigma_{z_{2}}\left[K Q_{x z_{2}}+G_{x z_{2}}\right] .
\end{aligned}
$$

Isolating those terms in the HJB equation involving the instantaneous investment control we find that the optimal investment policy solves

$$
\max _{I} I Q(X, S, \mathbf{Z})-I-\gamma I^{2} \Rightarrow I^{*}(X, S, \mathbf{Z})=\frac{Q(X, S, \mathbf{Z})-1}{2 \gamma}
$$

That is, investment is linear in the shadow value of capital $Q$. 
Next we note that since the HJB equation must hold pointwise, the terms scaled by $K$ must equate. Using this fact and rearranging terms we obtain an equation for pinning down the shadow value of capital $Q$ :

$$
\begin{aligned}
& (r+\delta+\lambda) Q(X, S, \mathbf{Z}) \\
= & \Pi_{S} X+\mu(\mathbf{Z}) X Q_{x}+\mu_{z_{1}} Q_{z_{1}}+\mu_{z_{2}} Q_{z_{2}}+\lambda Q\left(X, S^{\prime}, \mathbf{Z}\right) \\
& +\frac{1}{2} \sigma^{2} X^{2} Q_{x x}+\frac{1}{2} \sigma_{z_{1}}^{2} Q_{z_{1} z_{1}}+\frac{1}{2} \sigma_{z_{2}}^{2} Q_{z_{2} z_{2}} \\
& +\sigma_{z_{1}} \sigma_{z_{2}} Q_{z_{1} z_{2}}+X \sigma \sigma_{z_{1}} Q_{x z_{1}}+X \sigma \sigma_{z_{2}} Q_{x z_{2}}
\end{aligned}
$$

Since the dividend is linear in $X$ we conjecture the shadow value of capital is also linear in $X$. We then write the shadow value as follows:

$$
Q(X, S, \mathbf{Z})=X \Psi_{S}(\mathbf{Z})
$$

Substituting this into the preceding shadow value equation and simplifying we obtain

$$
\begin{aligned}
& {[r+\delta-\mu(\mathbf{Z})+\lambda] \Psi_{S}(\mathbf{Z}) } \\
= & \Pi_{S}+\left(\mu_{z_{1}}+\sigma \sigma_{z_{1}}\right) \frac{\partial}{\partial Z_{1}} \Psi_{S}(\mathbf{Z})+\left(\mu_{z_{2}}+\sigma \sigma_{z_{2}}\right) \frac{\partial}{\partial Z_{2}} \Psi_{S}(\mathbf{Z})+\lambda \Psi_{S^{\prime}}(\mathbf{Z}) \\
& +\frac{1}{2} \sigma_{z_{1}}^{2} \frac{\partial^{2}}{\partial Z_{1}^{2}} \Psi_{S}(\mathbf{Z})+\frac{1}{2} \sigma_{z_{2}}^{2} \frac{\partial^{2}}{\partial Z_{2}^{2}} \Psi_{S}(\mathbf{Z})+\sigma_{z_{1}} \sigma_{z_{2}} \frac{\partial^{2}}{\partial Z_{1} \partial Z_{2}} \Psi_{S}(\mathbf{Z})
\end{aligned}
$$

Next we conjecture there exist constants such that

$$
\Psi_{S}(\mathbf{Z})=Z_{1} \Psi_{S}^{1}+Z_{2} \Psi_{S}^{2} \Rightarrow Q(X, S, \mathbf{Z})=X\left[Z_{1} \Psi_{S}^{1}+Z_{2} \Psi_{S}^{2}\right]
$$

That is, we conjecture the shadow value of capital in each state represents a weighted average over macroeconomic beliefs. Effectively, $X \Psi_{S}^{n}$ represents the shadow value of capital in policy state $S$ from the perspective of a hypothetical investor who knows current instantaneous drift is $\mu_{n}$.

Substituting this conjecture into the preceding shadow value equation we obtain

$$
\begin{aligned}
& {[r+\delta-\mu(\mathbf{Z})+\lambda]\left[Z_{1} \Psi_{S}^{1}+Z_{2} \Psi_{S}^{2}\right] } \\
= & \Pi_{S}+\left(\mu_{z_{1}}+\sigma \sigma_{z_{1}}\right) \Psi_{S}^{1}+\left(\mu_{z_{2}}+\sigma \sigma_{z_{2}}\right) \Psi_{S}^{2}+\lambda\left[Z_{1} \Psi_{S^{\prime}}^{1}+Z_{2} \Psi_{S^{\prime}}^{2}\right] .
\end{aligned}
$$


Next we use the law of motion for beliefs in order to rewrite the preceding equation as

$$
\begin{aligned}
& {\left[r+\delta-Z_{1} \mu_{1}-Z_{2} \mu_{2}+\lambda\right]\left[Z_{1} \Psi_{S}^{1}+Z_{2} \Psi_{S}^{2}\right] } \\
= & \Pi_{S}+\left[p\left(f_{1}-Z_{1}\right)+Z_{1}\left(\left(1-Z_{1}\right) \mu_{1}-Z_{2} \mu_{2}\right)\right] \Psi_{S}^{1} \\
& +\left[p\left(f_{2}-Z_{2}\right)+Z_{2}\left(\left(1-Z_{2}\right) \mu_{2}-Z_{1} \mu_{1}\right)\right] \Psi_{S}^{2} \\
& +\lambda\left[Z_{1} \Psi_{S^{\prime}}^{1}+Z_{2} \Psi_{S^{\prime}}^{2}\right] .
\end{aligned}
$$

The preceding equation must hold for all possible beliefs including either extreme: $Z_{1}=1$ or $Z_{2}=1$. Using this fact we obtain the following two equations

$$
\begin{aligned}
& {\left[r+\delta-\mu_{1}+\lambda+p f_{2}\right] \Psi_{S}^{1}=\Pi_{S}+p f_{2} \Psi_{S}^{2}+\lambda \Psi_{S^{\prime}}^{1}} \\
& {\left[r+\delta-\mu_{2}+\lambda+p f_{1}\right] \Psi_{S}^{2}=\Pi_{S}+p f_{1} \Psi_{S}^{1}+\lambda \Psi_{S^{\prime}}^{2}}
\end{aligned}
$$

Of course, this condition must hold for either $S \in\{S 1, S 2\}$. Thus, we have the following lemma pinning down the shadow value of capital in the experimental economy.

Lemma In the experimental economy, the shadow value of capital is

$$
Q(X, S, \mathbf{Z})=X \Psi_{S}(\mathbf{Z})=X\left[Z_{1} \Psi_{S}^{1}+Z_{2} \Psi_{S}^{2}\right]
$$

where the shadow value constants solve the following linear system

$$
\begin{aligned}
& {\left[r+\delta-\mu_{1}+\lambda+p f_{2}\right] \Psi_{S 1}^{1}=\Pi_{S 1}+p f_{2} \Psi_{S 1}^{2}+\lambda \Psi_{S 2}^{1}} \\
& {\left[r+\delta-\mu_{2}+\lambda+p f_{1}\right] \Psi_{S 1}^{2}=\Pi_{S 1}+p f_{1} \Psi_{S 1}^{1}+\lambda \Psi_{S 2}^{2}} \\
& {\left[r+\delta-\mu_{1}+\lambda+p f_{2}\right] \Psi_{S 2}^{1}=\Pi_{S 2}+p f_{2} \Psi_{S 2}^{2}+\lambda \Psi_{S 1}^{1}} \\
& {\left[r+\delta-\mu_{2}+\lambda+p f_{1}\right] \Psi_{S 2}^{2}=\Pi_{S 2}+p f_{1} \Psi_{S 2}^{1}+\lambda \Psi_{S 1}^{2}}
\end{aligned}
$$


Figure 1: Shares Issued and Stock Price
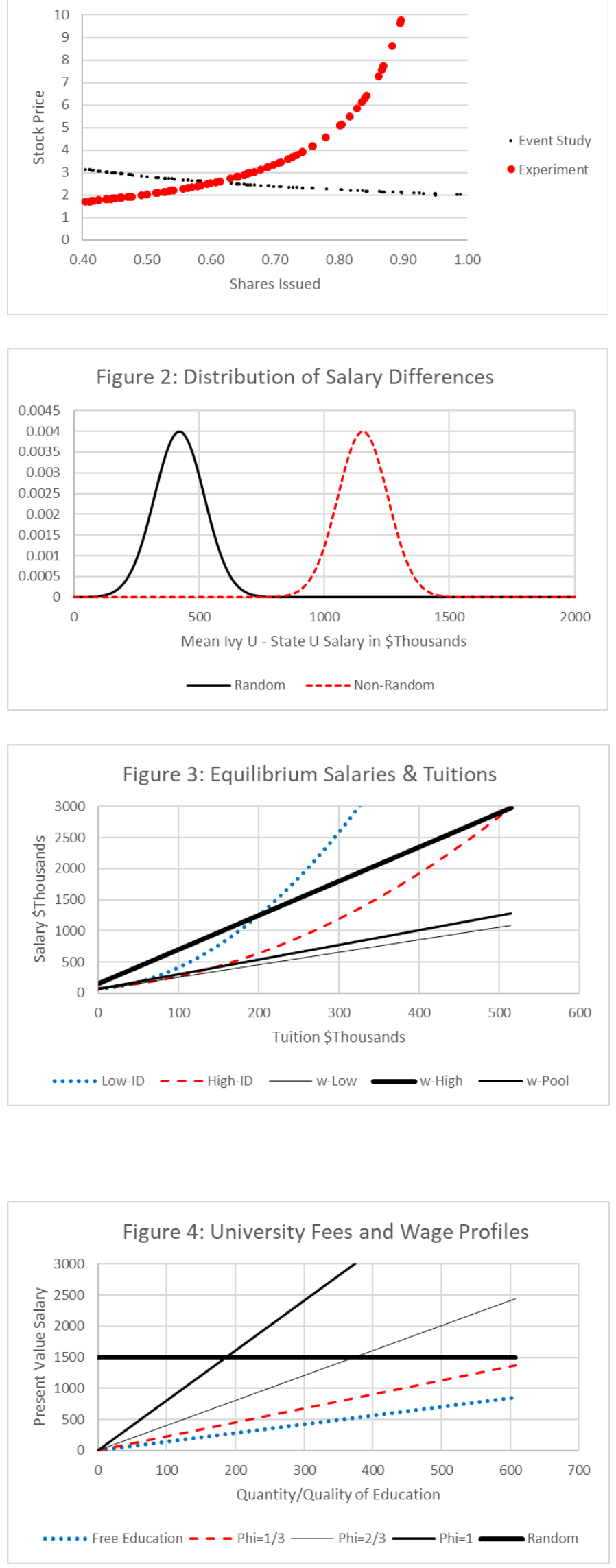